\title{
Sistema de Reconocimiento de Rostros usando Visión Estéreo
}

\author{
Elizabeth García-Rios, Enrique Escamilla-Hernández, Mariko Nakano-Miyatake y Héctor Pérez-Meana \\ Escuela Superior de Ingeniería Mecánica y Eléctrica, Instituto Politécnico Nacional. Av. Santa Ana 1000, \\ Col. San Francisco Culhuacán, 04430 México D.F. México (e-mail: (elizafiro@gmail.com, \\ zecmol@hotmail.com,mnakano@ipn.mx, hmperezm@ipn.mx)
}

Recibido Dic. 4, 2013; Aceptado Feb. 5, 2014; Versión final recibida Mar. 26, 2014

\section{Resumen}

Este artículo propone un sistema biométrico usando imágenes estéreo que permite incrementar la seguridad de los sistemas de reconocimiento de rostros convencionales, al poder discriminar entre imágenes de rostro reales en 3D y falsificadas en 2D, empleando la información proporcionada por las imágenes estéreo. Una vez que el sistema determina que la imagen de entrada es un rostro real en 3D, cada imagen estéreo es caracterizada e independientemente procesada usando un método de extracción de características $(\mathrm{EC})$ convencional. Seguidamente se emplea un método de fusión (MF) para combinar la información obtenida de ambas imágenes. Con el fin de determinar la combinación EC-MF más adecuada, se analizaron tres métodos de extracción de características y tres métodos de fusión. Los resultados experimentales obtenidos muestran que realizando la fusión de los datos extraídos mediante la transformación ondoleta discreta, antes de pasar a la etapa de clasificación, se obtienen los mejores resultados.

Palabras clave: reconocimiento de rostros, visión estéreo, niveles de fusión, PCA, transformada ondoleta discreta, funciones de Gabor

\section{Face Recognition system using Stereo Vision}

\begin{abstract}
This paper proposes a biometric system using stereo images to improve the security performance of conventional face recognition systems, by discriminating between $3 \mathrm{D}$ real and forged $2 \mathrm{D}$ faces images, using the tridimensional information provided by the stereo images. Once the system determines that the face under analysis is an authentic 3D image, each stereo image is characterized and independently processed using three feature extraction methods. Then a fusion method is required to combine the information of both images. To this end three fusion methods are analyzed to achieve a more robust face recognition system. The experimental results show that by performing the fusion of the data extracted by wavelet transform, before going to the classification step, better results are obtained.
\end{abstract}

Keywords: face recognition, stereo vision, fusion levels, PCA, wavelet transform, Gabor functions 


\section{INTRODUCCIÓN}

Los sistemas biométricos, los cuales emplean diversas características físicas o de comportamiento aprendido de las personas para su identificación, han sido un tópico de activa investigación durante los últimos años, tiempo en el cual se han desarrollado diversos sistemas tanto para la identificación de personas como para la verificación de su identidad (Jain y col, 2008; Ngugi y col., 2011). Entre los sistemas biométricos reportados hasta la fecha existen esquemas basados en características de la voz, del rostro, las huellas dactilares, en la firma autógrafa, la cadencia de tecleo, la forma de caminar, etc. (Gibbs, 2010). Entre ellos los sistemas basados en el reconocimiento de rostros son de los más utilizados debido a su naturaleza no-intrusiva, ya que en éstos la adquisición de los datos se lleva a cabo simplemente tomando una fotografía.

Adicionalmente, éste es el mecanismo más frecuentemente usado por las personas para reconocerse entre sí (Zhonghua y col. 2013; Vezzeti y Marcolin, 2012; Fookes y col. 2012). Durante los últimos años diversos esquemas de reconocimiento de rostros se han incorporado en diversos equipos comerciales, proporcionando porcentajes de reconocimiento superiores al $95 \%$ cuando operan en condiciones controladas. Sin embargo su funcionamiento se degrada considerablemente cuando operan en ambientes con iluminación no controlada, orientación variable del rostro con respecto a la cámara; así como cuando la imagen del rostro capturada contiene oclusiones significativas (Corcoran, 2011).

Otra de las limitaciones de los sistemas de reconocimiento de rostro actuales, la cual ha recibido menos atención que las anteriormente mencionadas, es el hecho de que el rostro es un objeto tridimensional y la gran mayoría de los sistemas desarrollados hasta la fecha, están basados en imágenes bidimensionales (Jin y Zhuojun, 2009; Faruqe y Hasan, 2000; Aguilar y col., 2009; Olivares y col. 2007). Esto permitiría engañar al sistema colocando frente a la cámara una fotografía de alta resolución de la persona cuya identidad se desea suplantar. Para evitar este problema, en este artículo se propone un sistema de reconocimiento de rostros empleando imágenes estéreo, con el fin de discriminar entre imágenes tridimensionales e imágenes bidimensionales, previniendo así el empleo de imágenes capturadas de una fotografía de la persona cuya identidad se pretende suplantar. Así, sí el sistema determina que la imagen capturada corresponde a una fotografía, el reconocimiento se detiene; mientras que en caso contrario se procedería a la extracción de características para el posterior reconocimiento de la imagen de entrada.

Una vez que el sistema determina que la imagen de entrada es auténtica, se procede a la caracterización de cada una de las imágenes estéreo, para lo cual se analizaron tres esquemas altamente eficientes: el análisis de componentes principales (PCA), (Turk, y Pentland, 1991; Kshirsagar y col. 2011), los filtros de Gabor (GF), (Aguilar y col., 2009; Jing y col. 2011); y la transformada ondoleta discreta (DWT por sus siglas en inglés), (Olivares y col. 2007; Santamaría y col. 2012; Rubio y col. 2012). Seguidamente, debido a que se obtiene un vector característico por cada imagen estéreo, con el fin de sacar el mayor provecho de los mismos, se realiza una fusión, ya sea del resultado obtenido de manera independiente de éstos o una fusión de los vectores característicos, para tomar la decisión final relativa a la identificación de persona bajo análisis, o la verificación de la identidad de la misma. Una vez procesados los vectores característicos, éstos se insertan a un esquema de clasificación para la toma de la decisión final.

\section{SISTEMA PROPUESTO}

El sistema de reconocimiento de rostros propuesto, mostrado en la figura 1, está constituido por dos etapas principales: la etapa de entrenamiento y la etapa de identificación o verificación de identidad del individuo. Aquí en la etapa de entrenamiento el sistema genera los modelos de las personas bajo análisis y los almacena en la base de datos, mientras que la etapa de identificación determina la identidad de la persona bajo análisis o verifica su identidad, empleando los modelos almacenados en la base de datos. Las siguientes sub secciones proporcionan un descripción de cada una de las etapas del sistema propuesto.

\section{Etapa de pre-procesamiento}

En esta etapa inicial se elimina el ruido aditivo que puede llegar a contaminar la imagen capturada, se segmenta la imagen y eventualmente, dependiendo del tamaño de la imagen capturada, se realiza una reducción de la dimensión de la imagen de entrada mediante una operación de filtrado pasa bajas, similar al realizado en la DWT, para facilitar el análisis de la misma. En caso de ser necesario, en esta etapa se podría también llevar a cabo la restauración de la imagen de entrada empleando esquemas de igualación del histograma para mejorar su calidad cuando ésta ha sido alterada debido a cambios en la iluminación (Olivares-Mercado y col., 2007). 


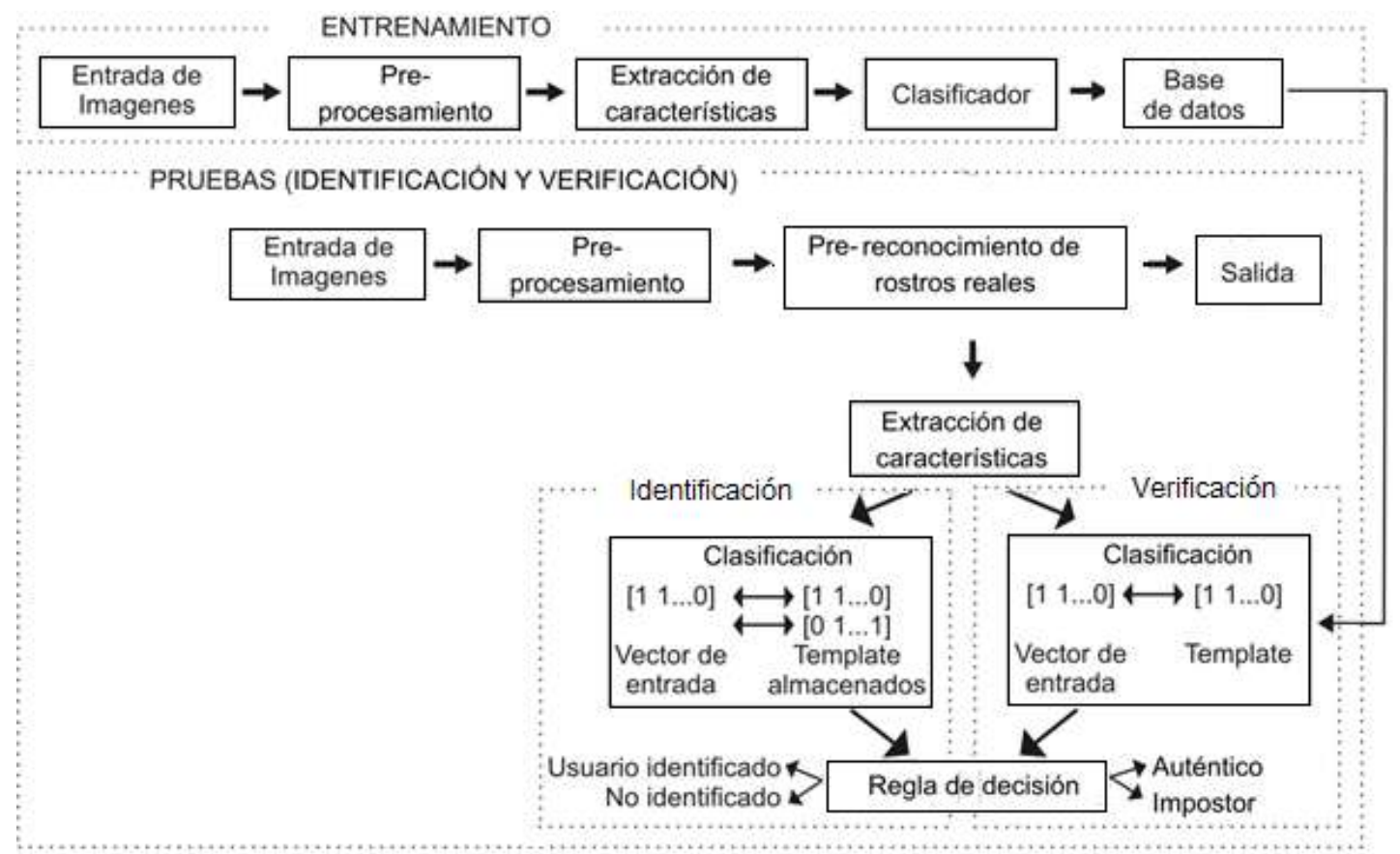

Fig. 1. Diagrama a bloques del sistema de reconocimiento de rostros.

Etapa de pre-reconocimiento de rostros reales.

La etapa de pre-reconocimiento, mostrada en la figura 2, intenta evitar la suplantación de identidad mediante el uso de las dos imágenes estéreo presentes a la entrada del sistema. A fin de determinar si las imágenes estéreo que se presentan a la entrada del sistema corresponden a un rostro real, tridimensional o una imagen en 2 dimensiones obtenida a partir de una fotografía, de alta resolución, perteneciente a una de las personas registradas en el sistema, se calcula la disparidad existente entre la imagen derecha e izquierda del rostro de entrada obtenidas usando una cámara estero. La idea principal detrás de este procedimiento es que la diferencia existente entre la imagen izquierda y derecha de un rostro real, figura 3 , al ser un volumen, es mayor que la diferencia obtenida cuando las imágenes de entrada corresponden de un objeto plano tal como se muestra en la figura 4.

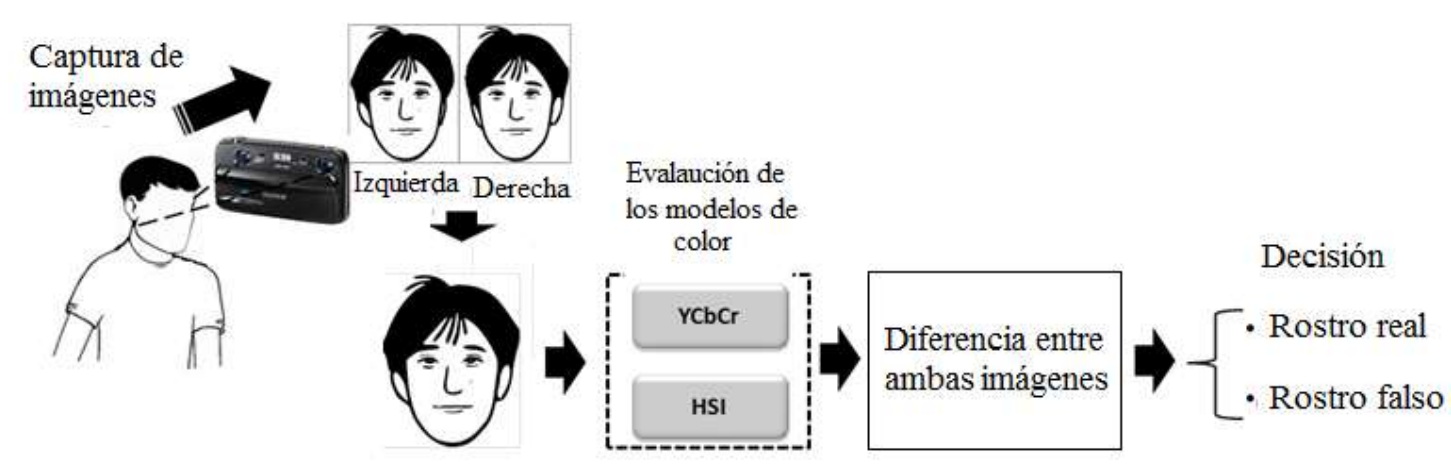

Fig. 2. Diagrama de la etapa de pre-reconocimiento.
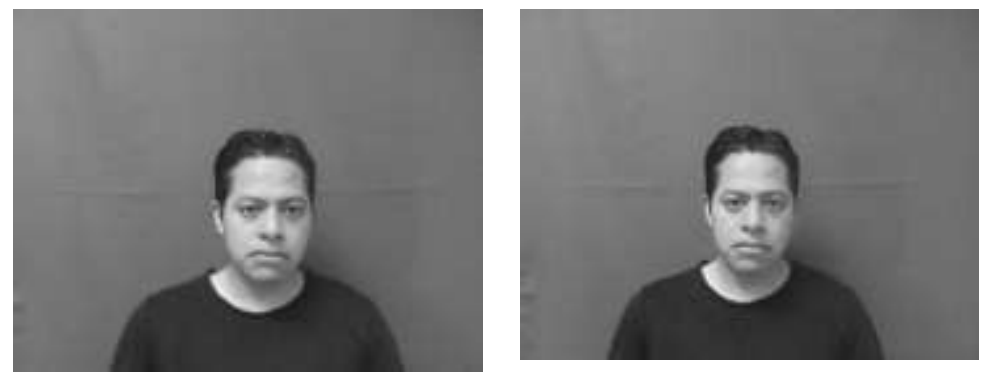

Fig. 3. Componentes izquierda y derecha de una imagen estéreo 


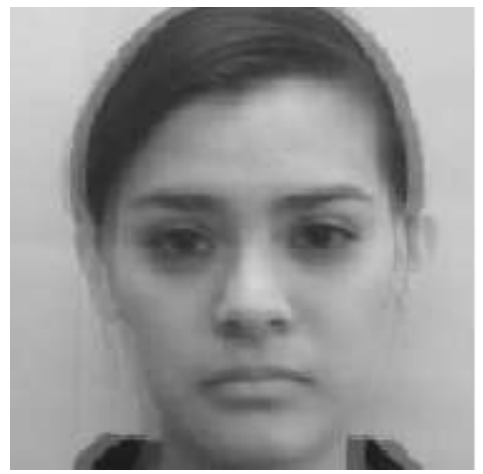

(a)

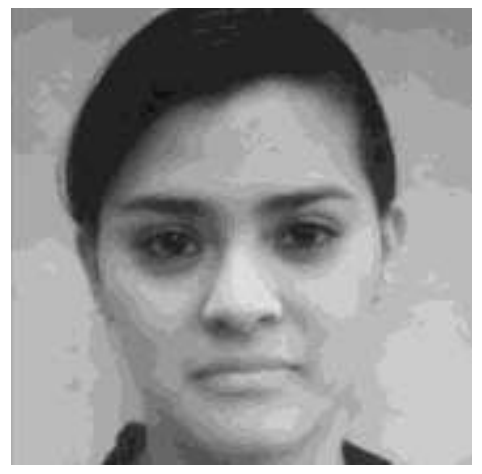

(b)

Fig. 4. Traslape existente entre las imágenes tomadas de (a) un rostro real (b) de una fotografía.

A continuación se detalla el procedimiento realizado en esta etapa. Cada una que las imágenes estero de entrada se pre-procesan como se mencionó anteriormente, se realiza la conversión de las mismas a los modelos de color YCbCr o HSI (Trussell y col. 2005; Marcial-Basilio y col. 2012) separándose en las componentes de intensidad o luminancia y cromas, que comprenden los modelos de color, conservándose únicamente las componente de intensidad (I), cuando se emplea el formato HSI, o las componentes de luminancia $(\mathrm{Y})$ cuando se emplea el formato $\mathrm{YCbCr}$. Una vez adquiridas las componentes ya sea I o Y, de imágenes de entrada las cuales son imágenes en escala de grises, se obtiene la diferencia existente entre las imágenes estéreo izquierda y derecha del rostro. Si las imágenes derecha e izquierda corresponden a un volumen, ambas imágenes presentan diferencias significativas entre si y no se empalman perfectamente, como se muestra en la figura 4 (a), dando como resultado una diferencia significativa en el valor de un número considerable de pixeles. Esta diferencia es mas evidente en los extremos de imagen del rostro como se puede ver en la figura 4 (a). Por otro lado cuando ambas imágenes corresponden a un objeto en 2-dimensiones, el empalme es casi perfecto, como se muestra en la figura 4(b). Tomando en cuenta lo anterior, para determinar si se trata de una imagen obtenida de un rostro real, se calcula el promedio de la diferencia absoluta entre la luminancia o intensidad de la imagen izquierda $l_{i}(m, n)$ y la imagen derecha $I_{d}(m, n)$, pixel a pixel, esto es:

$D_{p}=\frac{1}{M N} \sum_{m=0}^{M-1 N-1} \sum_{n=0}\left|I_{i}(m, n)-I_{d}(m, n)\right|$.

Aquí si $D_{p}$ es mayor que un umbral (Th) dado de antemano, se considera que la imagen corresponde a un rostro real, en cuyo caso ambas imágenes se insertan en la etapa de extracción de características (García y col. 2013). Por otro lado, si $D_{p}$ es menor que el umbral dado de antemano, el sistema determina que la imagen corresponde a un rostro falso, concluyendo en este momento el proceso de reconocimiento o verificación de identidad. Esta decisión se basa en el hecho de que en un par de imágenes estéreo tomadas directamente de un rostro real, la diferencia entre los valores de Luminancia o Intensidad de ambas imágenes es alto ya que existe diferencia relativamente grande entre el valor de los pixeles de ambas imágenes ubicados en la misma posición debido a la disparidad que presentan. Por otro lado, cuando las imágenes son tomadas de fotografía en 2-dimensiones la diferencia entre la Luminancia o Intensidad existente entre las imágenes derecha e izquierda es bajo, ya que no existe una diferencia apreciable entre el valor de los pixeles de ambas imágenes localizados en la misma posición. Con el fin de determinar un valor adecuado para el umbral se realizó una evaluación experimental en la cual se capturaron imágenes estéreo de 35 personas diferentes e imágenes estéreo de fotografías de cada una de estas 35 personas. Los resultados experimentales obtenidos muestran que una posible selección de los umbrales, Th, apropiados serían: $T h=0.05$ cuando se emplea la intensidad y $T h=0.025$ cuando se emplea la Luminosidad. (García y col. 2013). La figura 5 muestra las distribuciones de los valores de $D_{p}$, junto con los valores de $T h$.

\section{Extracción de características faciales.}

Una vez que la etapa de pre-reconocimiento determina que la imagen bajo análisis corresponde a un rostro real, se procede a la extracción de características usando ya sea: el Análisis de Componentes Principales (PCA), las funciones de Gabor (GF) o la DWT los cuales han sido empleados con éxito en diversos esquemas de reconocimiento de rostros. (Corcoran, 2011). 


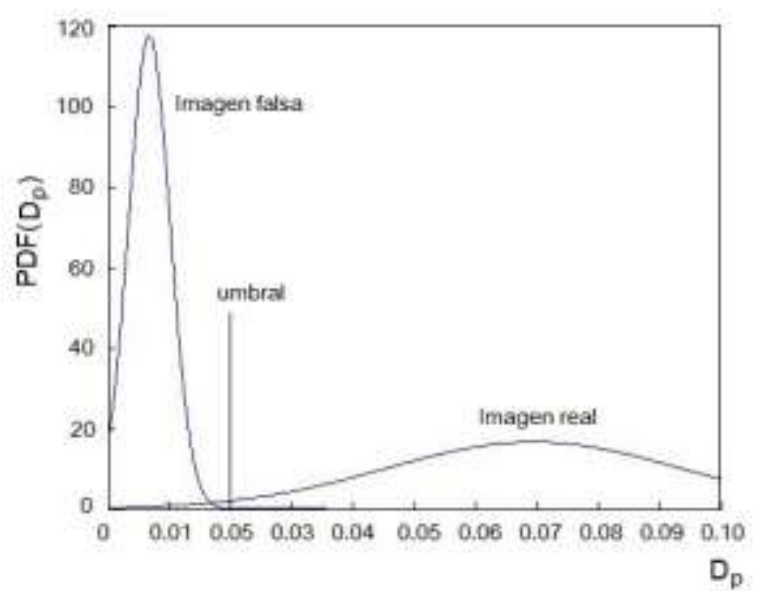

(a)

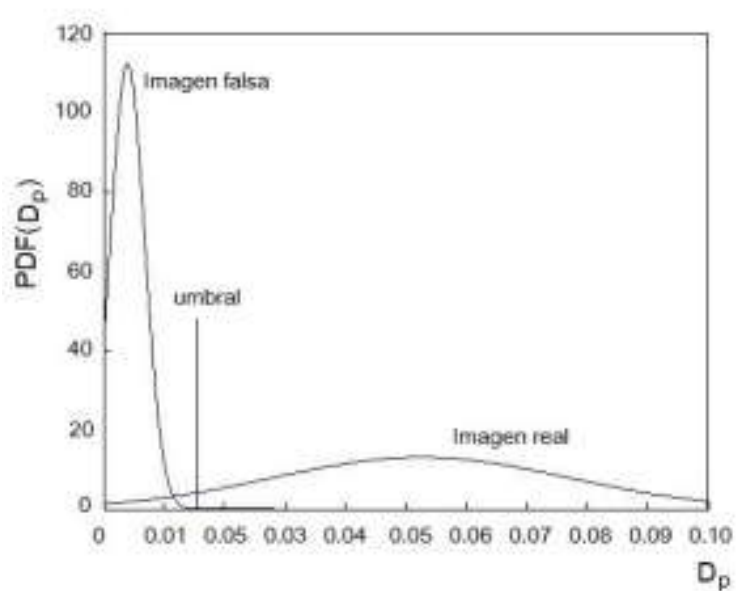

(b)

Fig. 5: Valores obtenidos de $D_{p}$ cuando se emplea la Luminosidad (Y) y la Intensidad (I); (a) empleando el formato $\mathrm{YCbCr}$ y (b) empleando el formato HSI.

\section{Extracción de características usando Análisis de Componentes Principales (PCA)}

Uno de los métodos más usados para reducir la dimensión de un problema de reconocimiento de patrones es el PCA (Aguilar y col., 2009; Faruqe y Hassan, 2009; Kshirsagar y col. 2011; Turk, y Pentland, 1991), el cual permite representar las imágenes bajo análisis, en una forma extremadamente compacta en comparación con el tamaño de las imágenes mismas. Con el fin de establecer las ecuaciones principales del PCA para llevar a cabo la caracterización de las imágenes de los rostros, considere un conjunto de M imágenes en niveles de gris, cada una de $L=N_{1} x N_{2}$ pixeles, las cuales se arreglan en una matriz de dimensión $L x M$. Seguidamente, con el fin de encontrar los vectores que mejor representan la distribución de imágenes del rostro bajo análisis, a cada imagen de entrenamiento, $\Gamma_{\mathrm{i}}, \mathrm{i}=1,2, \ldots, \mathrm{M}$, se le resta la imagen $\Psi$ la cual se obtiene promediando las $M$ imágenes de entrenamiento. De manera que se obtiene el conjunto de imágenes dado por (Aguilar y col., 2009; Faruqe y Hassan, 2009)

$\Phi_{\mathrm{i}}=\Gamma_{i}-\Psi \quad i=1,2 \ldots, M$.

Para reducir la dimensión de los vectores involucrados se puede emplear el método de descomposición en valores singulares (SVD) el cual establece que si $U_{k}$ y $\lambda_{k}$ son el k-ésimo vector propio y k-ésimo valor propio, respectivamente, de la matriz de covarianza $\mathrm{C}$, dada por

$$
C=\frac{1}{M} \sum_{k=1}^{M} \Phi_{k} \Phi_{k}^{T}=A A^{T}
$$

\section{Entonces}

$$
U_{k}^{T} C U_{k}=\lambda_{k}
$$

Multiplicando ambos lados por $U_{k}^{T}$ y usando la propiedad de ortonormalidad de los vectores propios, de la ecuación (3) obtenemos (Faruqe y Hassan, 2009; Kshirsagar y Col. 2011).

$$
A A^{T} U_{k}=\lambda_{k} U_{k}, \quad k=1,2,3, \ldots, M
$$

Seguidamente, multiplicando en ambos lados por la izquierda por $A^{T}$ se obtiene

$$
\begin{aligned}
& A^{T} A\left(A^{T} U_{k}\right)=\lambda_{k}\left(A^{T} U_{k}\right), \quad k=1,2,3, \ldots, M \\
& A^{T} A Y_{k}=\lambda_{k} Y_{k}, \quad k=1,2,3, \ldots, M
\end{aligned}
$$

Seguidamente, de la ecuaciones (5) y (7) se tiene que sí $U_{k}$ es el k-ésmo vector propio de $A A^{T}$ entonces $Y_{k}=A^{T} U_{k}$ es el k-ésimo vector propio de $A^{T} A$, estando ambos relacionados a través de la expresión 
$U_{k}=\frac{A Y_{k}}{\lambda_{k}}, \quad k=1,2,3, \ldots, M$

La importancia de esta reducción radica en el hecho de que mientras $A A^{T}$ puede llegar a tener hasta $L=N_{1} x N_{2}$ valores propios, donde $L$ es el número de pixeles de la imagen bajo análisis, $A^{T} A$ puede tener hasta $M$ valores propios, donde $M$ es el número de imágenes de entrenamiento, correspondiendo los $M$ valores propios de $A^{T} A$ a los $\mathrm{M}$ valores propios, mayores, de $A A^{T}$. Dado que en general $\mathrm{M}<<\mathrm{L}$, la reducción obtenida es significativa. Una vez que los $\mathrm{M}$ vectores propios han sido calculados, la imagen bajo análisis es proyectada en el espacio de rostro mediante una simple operación dada por (Turk y Pentland, 1991; Faruge y Hassan, 2009):

$w_{k}=U_{k}^{T}(\Gamma-\Psi), \quad k=1,2 \ldots, M$.

Finalmente los pesos que forman el vector $\Omega=\left[w_{1}, w_{2}, w_{3}, \ldots, w_{M}\right]$ describen la contribución de cada componente principal en el espacio de la imagen del rostro de entrada.

\section{Extracción de Características usando funciones de Gabor.}

Las funciones de Gabor en 2-dimensiones han sido ampliamente usadas para la caracterización de imágenes debido a que éstas guardan cierta relación con el sistema visual humano. Además de que presentan propiedades selectivas tanto de orientación como de frecuencia lo que les permite optimizar de manera conjunta la resolución en los dominios, tanto espacial como de frecuencia (Jones y Palmer, 1987). Las funciones de Gabor en 2-D están determinadas básicamente por cuatro parámetros, dos que expresan su localización en el dominio espacial $(x, y)$, otro que se relacionan con la frecuencia espacial de sintonía, $\omega=\pi / 2^{m}, m=1,2, . ., N_{\omega}-1$, y finalmente el último con la frecuencia espacial de orientación $\phi=n \pi / N_{\omega}, n=1,2, .$. , $N_{\phi}-1$. Así las funciones de Gabor se pueden expresar como:

$$
w\left(x_{n}, y_{n}, \omega_{m}, \phi\right)=g\left(x_{n}^{\prime}, y_{n}^{\prime}\right)\left(\cos \left[\omega_{m}\left(x_{n}^{\prime}+y_{n}^{\prime}\right)\right]+j \operatorname{sen}\left[\omega_{m}\left(x_{n}^{\prime}+y_{n}^{\prime}\right)\right]\right),
$$

donde

$$
g\left(x_{n}^{\prime}, y_{n}^{\prime}\right)=\left(\frac{1}{2 \pi \lambda \sigma^{2}}\right) \exp \left(\frac{\left(x_{n}^{\prime} / \lambda\right)^{2}+y_{n}^{\prime 2}}{\sigma^{2}}\right),
$$

$\sigma^{2}$ es el ancho de banda radial, $\lambda$ es el factor que determina la forma de la función Gaussiana y

$$
\left(x_{n}^{\prime}, y_{n}^{\prime}\right)=\left(x \cos \phi_{n}+y \operatorname{sen} \phi_{n}\right),\left(-x \operatorname{sen} \phi_{n}+y \cos \phi_{n}\right)
$$

es la posición del pixel $(\mathrm{x}, \mathrm{y})$ rotado un ángulo $\phi_{n}$.

Para caracterizar la imagen de un rostro empleando las funciones de Gabor, conservando el vector característico de la misma dimensión independientemente del tamaño de la imagen bajo análisis, inicialmente la imagen de entrada se divide en $M$ campos receptivos, cada uno de tamaño $\left(2 N_{x}+1\right)\left(2 N_{y}+1\right)$. Seguidamente se calcula el primer coeficiente de la correlación entre las $N_{\omega} N_{\phi}$, funciones de Gabor y cada uno de los $\mathrm{M}$ campos receptivos, donde $N_{\omega}$ es el número de frecuencias y $N_{\phi}$, es el número de ángulos, esto es:

$$
h(u, v)=\sum_{x=-N_{x}}^{N_{x}} \sum_{y=-N_{y}}^{N_{y}} I\left(x-c_{i}, y-d_{k}\right) w\left(x_{n}, y_{n}, \omega_{n}, \phi_{n}\right)
$$

Donde $c_{i}$ y $d_{k}$ denotan el pixel central del (i,j)-ésimo campo receptivo y $u=N_{y}(i-1)+k$, donde $k=1,2, \ldots, N_{x}$, y $v=N_{\omega}(m-1)+n$, con $n=1,2, \ldots, N_{\phi}$. La cantidad de parámetros obtenida por medio de la ecuación (13) es demasiado grande aun para valores relativamente pequeños de $N_{y}, N_{x}, N_{\omega}$ y $N_{\phi}$. Para reducir la cantidad de datos, conservando la información más importante, se calcula el valor promedio $h(u, v)$ en cada campo receptivo, el cual está dada por: 
$M(u)=\frac{1}{N_{\omega} N_{\phi}} \sum_{\nu=1}^{N_{\omega} N_{\phi}} h(u, v)$

El vector $M(u)$, dado por la ecuación (14), se emplea caracterizarlas imágenes de entrada.

Transformada discreta de ondoleta (DWT).

La DWT ha probado ser muy eficiente para llevara cabo la caracterización de imágenes de rostros mediante un análisis espacio-frecuencia (Alarcon y col. 2005; Martínez y col., 2010), donde la descomposición de la imagen de entrada se lleva a cabo usando simples operaciones de filtrado y decimación como se muestra en la figura 6. Aquí $I(n, m)$ denota la imagen de entrada, mientras que $I_{b b}^{k}(n, m), I_{b a}^{k}(n, m), I_{a b}^{k}(n, m), I_{a a}^{k}(n, m)$ denotan las componentes pasa bajas-bajas, pasa bajas-altas, pasa altas-bajas y pasa altas-altas, respectivamente obtenidas en el k-ésimo nivel de descomposición. Dado que las componentes pasa bajasbajas permiten compactar la mayor parte de la información de la imagen pese a que se descarten los detalles de la misma, únicamente las componentes pasa bajas-bajas son empleadas para caracterizar las imágenes bajo análisis. En la figura 7 muestra un ejemplo de los resultados obtenidos después de varios niveles de descomposición, empleando la DWT de Haar y de Daubechies de tercer orden (Martínez y col. 2010).

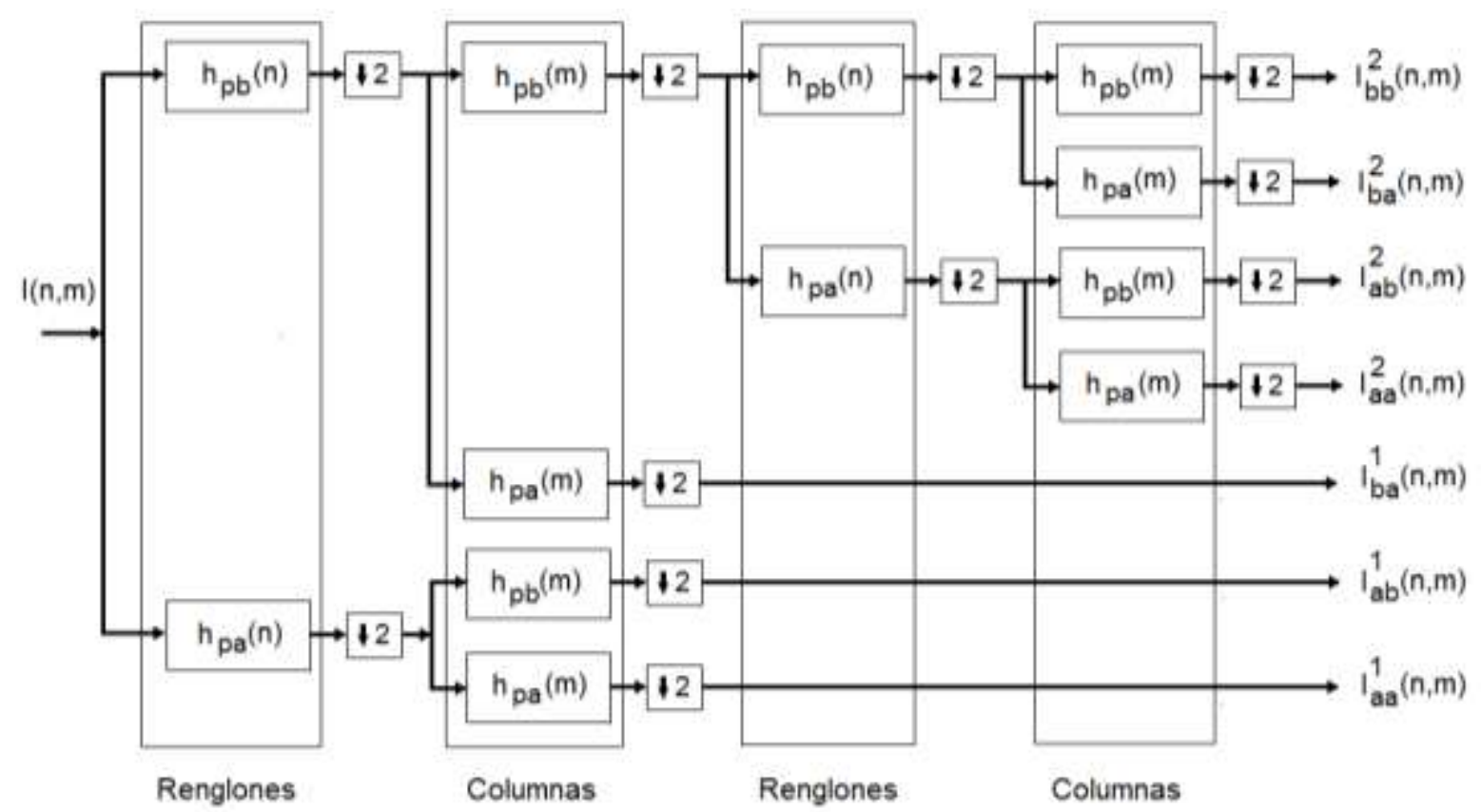

Fig. 6: Banco de filtros usados para obtener el segundo nivel de descomposición.

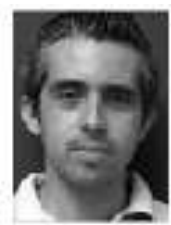

(a)

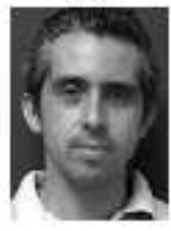

(e)

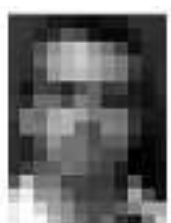

(b)

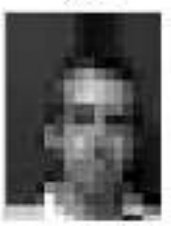

(f)

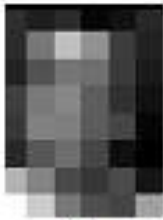

(c)

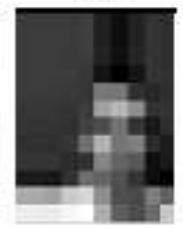

(g)

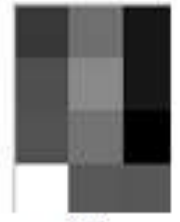

(d)

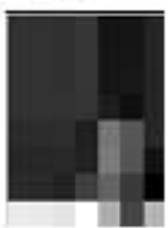

(h)

Fig. 7 Resultados obtenidos después de aplicar los primeros 4 niveles de descomposición a la imagen de entrada usando la DWT de Haar (a)-(d) y la DWT Daubechies (e)-(h) de tercer orden. 


\section{Etapa de clasificación.}

Después de obtener los vectores característicos en la etapa anterior, se lleva a cabo la identificación o verificación de la identidad de la persona bajo análisis, empleando una Maquina de Soporte Vectorial (SVM) (Yu y col. 2011; Chang y Lin, 2011) la cual, durante el entrenamiento, es responsable de generar un modelo para cada persona. Dado que el sistema propuesto puede ser empleado para verificar la identidad de una persona o para identifcar a la misma, la SVM puede ser entrenada de dos formas distintas. Si se requiere que el sistema verifique la identidad de una persona, la SVM es entrenada en forma tal que su salida se aproxime a uno cuando la persona bajo analisis es quien el o ella afirman ser, mientras su salida debe aproximarse a cero en caso contrario. Este trabajo se lleva a cabo usando solamente un modelo de la base de datos junto con un umbral dado. Por otro lado, cuando la operación a realizar es la identificación, la SVM es entrenada en forma tal que su valor de salida mayor correponda al modelo de la persona mas probable entre los que se encuentran en la base de datos, usando todos los modelos contenidos en ésta. En ambos casos, para entrenar la SVM se empleó el programa LIBSVM library escrito en Matlab (Chang y Lin, 2011) con un kernel polinomial del tipo (gamma* $u^{*} v+$ Coef0)-degree, donde coefo=1, gamma=1, cost $=100$. Los otros parámetros se fijaron con los valores de "default" sugeridos por los autores (Chang y Lin, 2011). Una vez obtenidos los modelos de las personas durante el entrenamiento, estos son almacenados en la base de datos para su uso durante los procesos ya sea la identificación o verificación.

Por su parte para llevar a cabo ya sea la verificación o identificación, los modelos almacenados en la base de datos se cargan en la SVM, obteniéndose el vector de salida de la misma cuando la entrada es el vector característico de la imagen bajo análisis. En el primer caso, el vector de salida que consiste de un solo elemento, se compara con un umbral para determinar si el vector de entrada corresponde o no, a la persona que dice ser. Por su parte, cuando el sistema opera como identificador, se obtiene el índice del valor máximo del vector de salida, el cual corresponderá a la persona identificada.

\section{Implementación de distintos niveles de fusión.}

Una vez que se lleva a cabo la caracterización de las imágenes derecha e izquierda de la persona bajo análisis, se requiere determinar la mejor manera de combinar o fusionar la información extraída de ellas, para lo cual se analizaron tres niveles diferentes para fusionar esta información. Los niveles de fusión analizados, son: fusión a nivel sensor, fusión a nivel características y fusión a nivel decisión. Cada nivel de fusión puede se puede considerar como un sistema constituido por los diferentes módulos que componen el sistema biométrico, descritos anteriormente. A continuación se describe cada uno de estos niveles de fusión.

\section{Fusión a nivel sensor.}

La fusión a nivel sensor se lleva a cabo después de que son obtenidas las imágenes de entrada, y antes de proceder a la caracterización de las mismas, mediante la combinación de la mitad izquierda de la imagen izquierda del rostro con la mitad derecha de la imagen del mismo lado; complementando de esta manera la información proporcionada por ambas imágenes estéreo.

Con esta finalidad, primeramente las imágenes son sometidas a la etapa de pre-procesamiento para la segmentación del rostro, seguidamente se verifica que estas correspondan a un rostro real por medio de la etapa de pre-reconocimiento. Seguidamente se segmentan las partes derecha e izquierda de ambas imágenes estéreo del rostro bajo análisis, llevándose a cabo finalmente la concatenación de la parte derecha de una con la izquierda de la otra, como se mencionó en el párrafo anterior, formándose así una nueva imagen del rostro conteniendo la información más relevante de cada imagen. Este proceso se muestra en la figura 8.
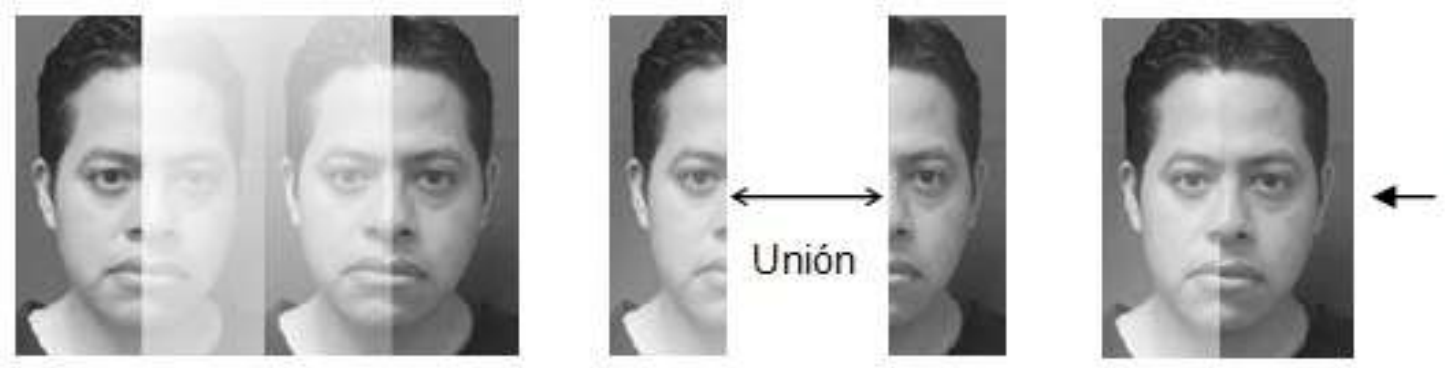

Imagen de

entrada

resultante

Fig. 8: Fusión de las imágenes izquierda y derecha. 
Una vez obtenida la imagen resultante se procede a insertarla en las etapas de extracción de características, clasificación y toma de decisión, mostrados en la figura 1, para su reconocimiento o verificación de identidad.

\section{Fusión a nivel de características}

En el procedimiento de fusión a nivel de características, la fusión de los datos se efectúa después de llevar a cabo la caracterización de las imágenes estéreo bajo análisis (García y col., 2013). A diferencia del nivel de fusión anterior, en este se caracteriza el par de imágenes estéreo, imagen izquierda e imagen derecha, por separado. Con esta finalidad, inicialmente introducen ambas imágenes a la etapa de pre-procesamiento para su segmentación, antes de pasarlas a la etapa de pre-reconocimiento para determinar si corresponden o no a un rostro real en 3D. En caso afirmativo, ambas imágenes se insertan en la etapa de extracción de características en la cual se obtienen los vectores característicos de cada imagen. Una vez caracterizada cada una de las imágenes se procede a la fusión los datos obtenidos la cual se realizó en dos formas distintas: La primera mediante el cálculo de la media aritmética, elemento por elemento, de los vectores característicos de ambas imágenes, dando como resultado un vector característico cuya longitud es igual a la cada uno de los vectores característicos individuales. La segunda opción consiste en la concatenación de ambos vectores característicos, lo que da como resultado un vector característico cuya dimensión es el doble de la dimensión de cada uno de los vectores característicos de cada una de las imágenes. La realización de cada uno de estos esquemas de fusión se muestra en la figura 9.

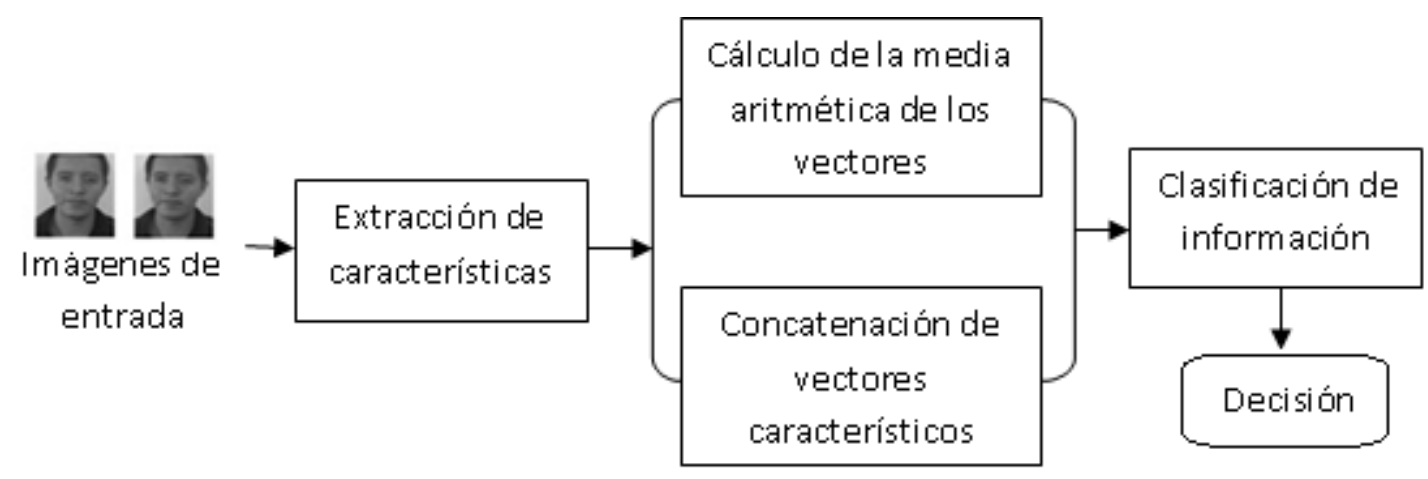

Fig. 9: Diagrama a bloques del nivel de fusión a nivel características

\section{Fusión a nivel decisión}

El último mecanismo de fusión es a partir del nivel de decisión, donde cada una de las imágenes que componen la imagen estéreo es procesada de manera independiente, mediante las etapas descritas con anterioridad. Inicialmente pasan a la etapa de pre-procesamiento, pasando seguidamente a la etapa de prereconocimiento para determinar si corresponden a un rostro real. Seguidamente se procede a la extracción de los vectores característicos de cada una de las imágenes, los cuales se insertan separadamente en dos etapas de identificación o verificación de identidad, independientes entre sí, en donde cada una de las cuales toma una decisión acerca de la identidad de la persona bajo análisis. Finalmente se aplica la fusión de los dos resultados obtenidos. Aquí el sistema determina que la verificación de identidad es positiva solamente si ambas etapas determinan que la persona bajo análisis es quien dice ser. Por otro lado, si se requiere que el sistema realice una operación de identificación, se promedian elemento a elemento los vectores de salida de ambas etapas de clasificación y se considera como la persona identificada aquella que corresponde al índice del vector de salida resultante cuyo valor promedio es máximo.

\section{EVALUACION DEL SISTEMA.}

Para llevar a cabo la evaluación del sistema y tomando en cuenta que no existe, hasta donde los autores conocen, una base de datos estandarizada para reconocimiento de rostro usando visión estéreo, se construyó una usando una cámara "Fujifilm FinePix® real 3D dual" de 10 Mega pixeles. La base construida consiste de 60 personas diferentes con 15 imágenes estéreo por cada persona, de las cuales 10 imágenes de cada persona se usaron para el entrenamiento del sistema y 5 para la realización de pruebas de identificación y verificación. Así, el número total de imágenes contenidas en esta base de datos es de 1800 imágenes, capturadas en un ambiente con iluminación y fondo controlado. La figura 10 muestra algunas de las imágenes que conforman esta base de datos. 

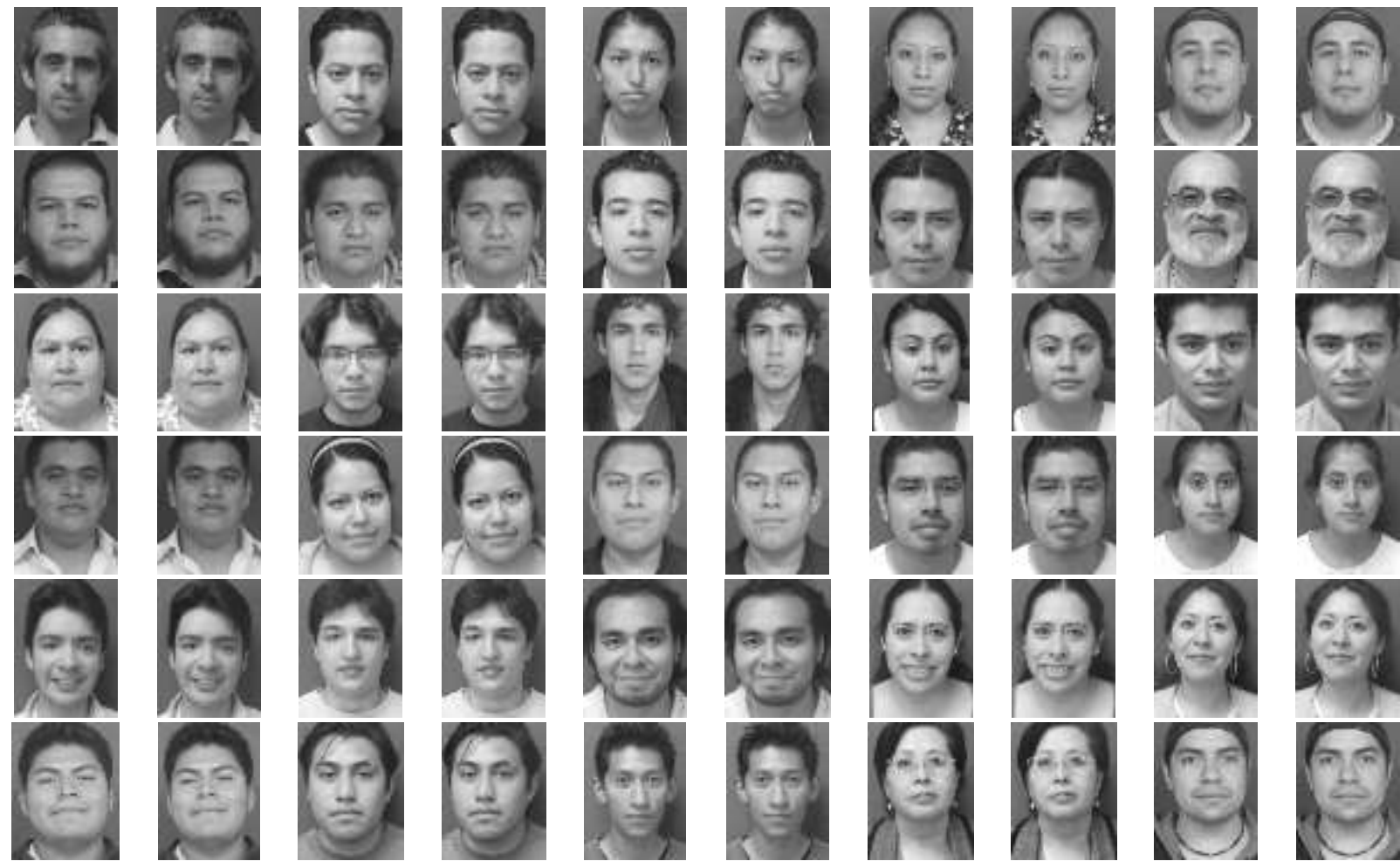

Fig. 10. Ejemplos de imágenes contenidas en la base de datos.

Para llevar a cabo la extracción de características, usando los filtros de Gabor, cada una de las imágenes de entrada fueron divididas en 108 campos receptivos, a los cuales se les aplicaron 54 funciones de Gabor por cada campo, con 6 frecuencias espaciales dadas por $\omega=\pi / 2^{m}, m=1,2, . ., 6 ;$ y 9 ángulos de orientación $\phi=n \pi / 9, n=1,2, . ., 8$, generándose un vector característico con 108 elementos, independientemente del tamaño de las imágenes de entrada. Por su parte cuando se empleó la DWT, tanto de Haar como de Daubechies, para la caracterización de las imágenes de entrada, se utilizaron 4, 5 y 6 niveles de descomposición. En estos casos los vectores de entrada dependen del tamaño de las imágenes de entrada, siendo estos iguales a $\left(N_{x} / 2^{d}\right) \times\left(N_{y} / 2^{d}\right)$, donde $N_{x} x N_{y}$ es el tamaño de la imagen original. Finalmente cuando se empleo el método de PCA para caracterizar las imágenes de entrada, la dimensión del vector característico depende del número de imágenes de entrenamiento, siendo en este caso igual 600 . Por su parte para llevar a cabo la evaluación de la etapa de pre-reconocimiento usada para determinar si el rostro es real o falso, se construyó una base de datos con 50 imágenes, 35 fotografías tomadas directamente del rostro, correspondientes a la base de datos mencionada anteriormente y 15 fotografías tomadas de otras fotografías de las mismas personas.

Tabla 1: Porcentajes de identificación

\begin{tabular}{|c|c|c|c|c|c|c|c|c|c|}
\hline \multirow{2}{*}{\multicolumn{2}{|c|}{ Niveles de Fusión }} & \multirow[b]{2}{*}{$P C A$} & \multirow[b]{2}{*}{ Gabor } & \multicolumn{3}{|c|}{ Haar } & \multicolumn{3}{|c|}{ Daubechies de orden 3} \\
\hline & & & & Nivel 4 & Nivel 5 & Nivel 6 & Nivel 4 & Nivel 5 & Nivel 6 \\
\hline \multicolumn{2}{|c|}{ Fusión a nivel sensor } & 99.22 & 99.50 & 99.55 & 99.55 & 99.66 & 99.77 & 99.88 & 99.77 \\
\hline \multirow{2}{*}{ Fusión a nivel características } & Media aritmética & 99.59 & 99.76 & 99.55 & 99.55 & 99.66 & 99.66 & 99.88 & 99.55 \\
\hline & Concatenación & 99.78 & 99.82 & 99.55 & 99.55 & 99.77 & 99.77 & 99.88 & 99.55 \\
\hline \multicolumn{2}{|c|}{ Fusión a nivel decisión } & 99.40 & 99.50 & 99.55 & 99.55 & 99.55 & 99.66 & 99.88 & 99.55 \\
\hline \multicolumn{2}{|c|}{ Soló parte derecha } & 99.46 & 99.51 & 99.55 & 99.55 & 99.55 & 99.66 & 99.88 & 99.77 \\
\hline \multicolumn{2}{|c|}{ Soló parte izquierda } & 99.50 & 99.52 & 99.55 & 99.55 & 99.55 & 99.77 & 99.88 & 99.55 \\
\hline
\end{tabular}


La Tabla 1 muestra el comportamiento del sistema cuando se efectúa el proceso de identificación usando los tres métodos de fusión descritos anteriormente. En todos los casos, los vectores característicos son obtenidos mediante las funciones de Gabor, el PCA, así como la DWT tanto de Haar como de Daubechies de orden 3. Los resultados obtenidos muestran que los cuatro esquemas se comportan de manera similar, difiriendo en menos de un punto porcentual entre ellos. Es importante hacer notar que en todos los casos los resultados obtenidos, cuando el sistema se evalúa usando solo una componente de las imágenes estéreo, son bastante cercanos a aquellos obtenidos en los cuales se emplean ambas imágenes.

Posteriormente se llevaron a cabo las pruebas de verificación de identidad, en las cuales el sistema debe determinar si la persona bajo análisis es quien dice ser. Con esta finalidad, inicialmente se llevó a cabo la evaluación del sistema omitiendo la etapa de pre-reconocimiento, obteniéndose los resultados mostrados en la Tabla 2. Seguidamente se repitió la evaluación del sistema incluyendo la etapa de pre-reconocimiento, obteniéndose en este caso los resultados mostrados en la Tabla 3. Los resultados mostrados en las Tablas 2 y 3 muestran que el sistema propuesto, empleando la fusión de las características de ambas imágenes estéreo, presenta un buen funcionamiento, ya que aún si usar la etapa de pre-reconocimiento solo entre el $6 \%$ y $10 \%$ de las imágenes falsas son aceptadas como verdaderas, mientras que usando la etapa de prereconocimiento la falsa aceptación se reduce a menos del $0.06 \%$ cuando se presentan imágenes falsas a la entrada del sistema.

Tabla 2: Porcentajes de verificación de identidad sin usar la etapa de pre-reconocimiento.

\begin{tabular}{|c|c|c|c|c|c|c|c|c|c|}
\hline \multirow{2}{*}{\multicolumn{2}{|c|}{ Niveles de Fusión }} & \multirow{2}{*}{$P C A$} & \multirow{2}{*}{ Gabor } & \multicolumn{3}{|c|}{ Haar } & \multicolumn{3}{|c|}{ Daubechies de orden 3} \\
\hline & & & & Nivel 4 & Nivel 5 & Nivel 6 & Nivel 4 & Nivel 5 & Nivel 6 \\
\hline \multicolumn{2}{|c|}{ Fusión a nivel sensor } & 5.55 & 5,88 & 5,88 & 7,84 & 7,84 & 7,84 & 7,84 & 11,76 \\
\hline & Media aritmética & 5.61 & 5,88 & 5,88 & 7,84 & 9,8 & 7,84 & 7,84 & 9,8 \\
\hline Fusión a nivel características & Concatenación & 5.53 & 5,88 & 5,88 & 7,84 & 9,8 & 9,8 & 7,84 & 9,8 \\
\hline \multicolumn{2}{|c|}{ Fusión a nivel decisión } & 5.49 & 5,88 & 5,88 & 7,84 & 9,8 & 7,84 & 7,84 & 9,8 \\
\hline \multicolumn{2}{|c|}{ Sólo parte derecha } & 5.45 & 5,88 & 5,88 & 7,84 & 9,8 & 7,84 & 7,84 & 9,8 \\
\hline \multicolumn{2}{|c|}{ Sólo parte izquierda } & 5,34 & 7,84 & 5,88 & 7,84 & 7,84 & 7,84 & 9,8 & 11,76 \\
\hline
\end{tabular}

Tabla 3: Porcentajes de verificación de identidad usando la etapa de pre-reconocimiento.

\begin{tabular}{|c|c|c|c|c|c|c|c|c|c|}
\hline \multirow{2}{*}{\multicolumn{2}{|c|}{ Niveles de Fusión }} & \multirow{2}{*}{$P C A$} & \multirow{2}{*}{ Gabor } & \multicolumn{3}{|c|}{ Haar } & \multicolumn{3}{|c|}{ Daubechies de orden 3} \\
\hline & & & & Nivel 4 & Nivel 5 & Nivel 6 & Nivel 4 & Nivel 5 & Nivel 6 \\
\hline \multicolumn{2}{|c|}{ Fusión a nivel sensor } & 0.02 & 0.02 & 0.02 & 0.04 & 0.04 & 0.04 & 0.04 & 0.06 \\
\hline \multirow{2}{*}{ Fusión a nivel características } & Media aritmética & 0.02 & 0.02 & 0.02 & 0.04 & 0.04 & 0.04 & 0.04 & 0.05 \\
\hline & Concatenación & 0.02 & 0.02 & 0.02 & 0.04 & 0.04 & 0.04 & 0.04 & 0.05 \\
\hline \multicolumn{2}{|c|}{ Fusión a nivel decisión } & 0.02 & 0.02 & 0.02 & 0.04 & 0.04 & 0.04 & 0.04 & 0.05 \\
\hline \multicolumn{2}{|c|}{ Sólo parte derecha } & 0.02 & 0.02 & 0.02 & 0.04 & 0.04 & 0.04 & 0.04 & 0.05 \\
\hline \multicolumn{2}{|c|}{ Sólo parte izquierda } & 0.02 & 0.04 & 0.02 & 0.04 & 0.04 & 0.04 & 0.05 & 0.06 \\
\hline
\end{tabular}

Las Tablas 4-6 muestran los resultados obtenidos cuando se requiere que el sistema verifique la identidad de las personas cuyas imágenes reales se presentan a su entrada. En la Tabla 4 muestra el funcionamiento del sistema propuesto cuando se emplea el PCA y las funciones de Gabor para llevar a cabo la extracción de características. En la Tabla 5 se muestran los resultados obtenidos cuando se emplea la DWT de Haar, mientras que en la Tabla 6 se muestran los resultados obtenidos usando la DWT de Daubechies de orden 3. En todos los casos se presentan, además del porcentaje de verificación, el porcentaje de falsa aceptación (FAR) y el porcentaje de falso rechazo (FRR) proporcionado por el sistema propuesto. 
Tabla 4: Porcentajes de verificación usando el método de PCA y las funciones de Gabor como extractores de características

\begin{tabular}{|c|c|c|c|c|c|c|c|}
\hline \multirow{2}{*}{ Niveles de fusión } & \multicolumn{3}{c|}{$P C A$} & \multicolumn{3}{c|}{ Gabor } \\
\cline { 3 - 9 } \multicolumn{2}{|c|}{} & $\%$ FAR & $\%$ FRR & $\%$ Verificación & $\%$ FAR & $\%$ FRR & $\%$ Verificación \\
\hline \multicolumn{2}{|c|}{ Fusión a nivel sensor } & 0.92 & 0.78 & 98.30 & 1,857 & 0,786 & 97,357 \\
\hline \multirow{2}{*}{ Fusión a nivel características } & Media aritmética & 0.59 & 0.31 & 99.10 & 2,429 & 0,786 & 96,785 \\
\cline { 2 - 9 } & Concatenación & 0.86 & 0.27 & 98.87 & 1,857 & 0,786 & 97,357 \\
\hline \multicolumn{2}{|c|}{ Fusión a nivel decisión } & 0.77 & 0.29 & 98.94 & 2,429 & 0,786 & 96,786 \\
\hline \multicolumn{2}{|c|}{ solo parte derecha } & 0.62 & 0.17 & 99.21 & 2,071 & 0,786 & 97,143 \\
\hline \multicolumn{2}{|c|}{ solo parte izquierda } & 0.65 & 0.23 & 99.12 & 1,786 & 0,714 & 97,5 \\
\hline
\end{tabular}

Tabla 5: Porcentajes de verificación usando trasformada de Haar como extractor de características.

\begin{tabular}{|c|c|c|c|c|c|c|c|c|c|c|}
\hline \multirow{2}{*}{\multicolumn{2}{|c|}{ Nivel de fusión }} & \multicolumn{3}{|c|}{ Nivel 4} & \multicolumn{3}{|c|}{ Nivel 5} & \multicolumn{3}{|c|}{ Nivel 6} \\
\hline & & $\begin{array}{l}\% \\
\text { FAR } \\
\end{array}$ & $\begin{array}{l}\% \\
\text { FRR }\end{array}$ & $\begin{array}{l}\% \\
\text { Verif }\end{array}$ & $\begin{array}{l}\% \\
\text { FAR }\end{array}$ & $\begin{array}{l}\% \\
\text { FRR }\end{array}$ & $\begin{array}{l}\% \\
\text { Verif }\end{array}$ & $\begin{array}{l}\% \\
\text { FAR }\end{array}$ & $\begin{array}{l}\% \\
\text { FRR }\end{array}$ & $\begin{array}{l}\% \\
\text { Verif }\end{array}$ \\
\hline \multicolumn{2}{|c|}{ Fusión a nivel Sensor } & 2,429 & 0,786 & 96,785 & 2,571 & 0,714 & 96,715 & 3,143 & 0,714 & 96,143 \\
\hline \multirow{2}{*}{$\begin{array}{l}\text { Fusión a nivel } \\
\text { características }\end{array}$} & $\begin{array}{c}\text { Media } \\
\text { aritmética }\end{array}$ & 2,429 & 0,786 & 96,785 & 2,571 & 0,714 & 96,715 & 3,286 & 0,643 & 96,071 \\
\hline & Concatenación & 2,143 & 0,786 & 97,071 & 2,286 & 0,714 & 97,000 & 3,214 & 0,643 & 96,143 \\
\hline \multicolumn{2}{|c|}{ Fusión a nivel decisión } & 2,429 & 0,786 & 96,786 & 3,214 & 0,714 & 96,071 & 3,786 & 0,643 & 95,571 \\
\hline \multicolumn{2}{|c|}{ solo parte derecha } & 2,429 & 0,786 & 96,786 & 2,571 & 0,714 & 96,714 & 3,286 & 0,643 & 96,071 \\
\hline \multicolumn{2}{|c|}{ solo parte izquierda } & 2,000 & 0,786 & 97,214 & 2,714 & 0,714 & 96,571 & 3,143 & 0,714 & 96,143 \\
\hline
\end{tabular}

Tabla 6: Porcentajes de verificación usando las funciones de Daubechies como extractor de características

\begin{tabular}{|c|c|c|c|c|c|c|c|c|c|c|}
\hline \multirow{2}{*}{\multicolumn{2}{|c|}{ Niveles de fusión }} & \multicolumn{3}{|c|}{ Nivel 4} & \multicolumn{3}{|c|}{ Nivel 5} & \multicolumn{3}{|c|}{ Nivel 6} \\
\hline & & $\begin{array}{l}\% \\
F A R\end{array}$ & $\begin{array}{l}\% \\
F R R\end{array}$ & $\begin{array}{l}\% \\
\text { Verif }\end{array}$ & $\begin{array}{l}\% \\
F A R\end{array}$ & $\begin{array}{l}\% \\
F R R\end{array}$ & $\begin{array}{l}\% \\
\text { Verif }\end{array}$ & $\begin{array}{l}\% \\
F A R\end{array}$ & $\begin{array}{l}\% \\
F R R\end{array}$ & $\begin{array}{l}\% \\
\text { Verif }\end{array}$ \\
\hline \multicolumn{2}{|c|}{ Fusión a nivel sensor } & 2,357 & 0,714 & 96,929 & 2,929 & 0,714 & 96,357 & 3,286 & 0,571 & 96,143 \\
\hline \multirow{2}{*}{$\begin{array}{l}\text { Fusión a nivel } \\
\text { características }\end{array}$} & $\begin{array}{c}\text { Media } \\
\text { aritmética }\end{array}$ & 2,429 & 0,714 & 96,857 & 2,929 & 0,714 & 96,357 & 3,286 & 0,643 & 96,071 \\
\hline & Concatenación & 2,143 & 0,643 & 97,214 & 2,929 & 0,714 & 96,357 & 3,571 & 0,643 & 95,786 \\
\hline \multicolumn{2}{|c|}{ Fusión a nivel decisión } & 2,5 & 0,714 & 96,786 & 2,929 & 0,714 & 96,357 & 3,286 & 0,643 & 96,071 \\
\hline \multicolumn{2}{|c|}{ solo parte derecha } & 2,429 & 0,714 & 96,857 & 2,928 & 0,714 & 96,357 & 3,286 & 0,643 & 96,071 \\
\hline \multicolumn{2}{|c|}{ solo parte izquierda } & 2,357 & 0,714 & 96,929 & 2,786 & 0,643 & 96,571 & 3,286 & 0,571 & 96,143 \\
\hline
\end{tabular}

\section{CONCLUSIONES}

Este trabajo propone un sistema de reconocimiento de rostros, usando visión estéreo, el cual permite evitar la suplantación de identidad. Este proceso se lleva a cabo en la etapa de pre-reconocimiento, estimando el promedio de la diferencia absoluta de la luminosidad o Intensidad, presente entre los pixeles las dos imágenes estéreo. Una vez capturadas las dos imágenes estéreo el sistema inicialmente se verifica si la 
imagen del rostro bajo análisis corresponde a un rostro real o a una fotografía, antes de proceder a llevar a cabo el reconocimiento o verificación de la identidad de la persona bajo análisis. Una vez que el sistema ha verificado que la imagen corresponde a un rostro real, se procede a la caracterización de las dos imágenes estéreo mediante el uso, ya sea de las funciones de Gabor, PCA o de la DWT. Seguidamente, una vez obtenido el vector característico de las imágenes estéreo, se emplea uno de los distintos niveles de fusión con el fin combinar de forma adecuada los vectores característicos obtenidos a partir de ambas imágenes.

Los resultados experimentales, usando la máquina de soporte vectorial (SVM) como clasificador, muestran que los tres niveles de fusión analizados, en general, presentan resultados altamente satisfactorios con los cuatro métodos de extracción de características usados. Sin embargo, el método de fusión a nivel características cuando se emplean los filtros de Gabor para llevar a cabo la extracción de características, mostró ser ligeramente superior a los demás, cuando el sistema es usado como identificador. Mientras que cuando el sistema opera como verificador los resultados obtenidos usando el PCA son ligeramente superiores a los demás. Por otro lado, los resultados obtenidos muestran también que cuando la entrada es bidimensional, usando la etapa de pre-reconocimiento, el sistema propuesto proporciona un porcentaje de falsa aceptación (FAR) inferior al $0.06 \%$, mientras que cuando el sistema trabaja sin la etapa de prereconocimiento, éste es porcentaje se incrementa a cerca del $8 \%$. Esto resalta la importancia del esquema de pre-reconocimiento propuesto, ya que permite reducir significativamente el porcentaje de falsa aceptación del sistema, como se muestra en las Tabla 2 y 3.

\section{AGRADECIMIENTOS}

Los autores agradecen al Consejo Nacional de Ciencia y Tecnología (CONACyT) y al Instituto Politécnico Nacional por el apoyo otorgado para la realización de este trabajo.

\section{REFERENCIAS}

Aguilar Torres G., Toscano Medina K., Sanchez Perez G., Nakano Miyatake M. y Pérez Meana H., Eigenface-Gabor algorithm for feature extraction in face recognition, International Journal of Computers,3(1), pp. 20-30, (2009).

Alarcon V., Guerrero L., Rodriguez A y Rosas L., Analisis de trafico autosimilar en redes de comunicación usando ondoletas (wavelets), Revista Información Tecnológica, 16(2) pp. 62-66, (2005).

Chang, C. y Lin, C., LIBSVM: A Library for Support Vector Machines. ACM T Intel Syst Tec, (2011).

Chun-yang L., Dao-zheng H., y Chang-an L., Study on moving object detection based on RGB color model, Advanced Materials Research 710, pp. 700 - 703, (2013).

Corcoran P., Reviews, Refinements and New Ideas in Face Recognition, Intech Publisher, Croatia, (2011)

Fookes C., Lin F., Chandran V. y Sridharan S., Evaluation of image resolution and super-resolution on face recognition performance, Journal of Visual Communication and Image Representation, 23 (1), pp. 75-93, (2012).

Faruqe O. y Hasan A., Face Recognition Using PCA and SVM, Anti-counterfeiting, Segurity and Identification in Communication, pp. 97-101, (2009).

García E., Escamilla E. Aguilar Torres G., Jacobo O., Nakano M. y Perez-Meana H., Multibiometric face recognition using level fusión, NAUN International Journal of Computers, 7(3) pp. 99-108 (2013).

Gibbs M., Biometrics: Body Odor Authentication Perception and Acceptance, ACM SIGCAS Computers and Society,40(4), pp. 16-24,(2010).

Jain A, Flynn P. y Ross A, Handbook of Biometrics, Springer Science+Business Media, LLC (2008).

Jing J., Zhang H. y Li P., Improved Gabor filters for textile defect detection, Procedia Engineering,15, pp. $5010-5014,(2011)$.

Jin R. y Zhuojun D., Face Recognition based on Multi-scale Singular Value Features, WSEAS Trans. on Computers, 8(1) pp. 143-152(2009). 
Jones J. y Palmer L., An evaluation of the two-dimensional Gabor Filter model of simple receptive fields in cat striate cortex, J. Neurophysiology, 58,pp. 1233-1258 (1987).

Marcial-Basilio J., Aguilar-Torres G., Sánchez-Pérez G., Toscano-Medina K. y Pérez-Meana H., Novedosa técnica para la detección de imágenes pornográficas empleando modelos de color HSV y YCbCr, Revista Facultad de Ingeniería, 64, pp. 79 - 90, (2012).

Kshirsagar V., Baviskar M. y Gaikwad M., Face Recognition Using Eigenfaces, International Conference on Computer Research and Development,pp. 302-306 (2011).

Martinez A., Pineda Z., y Ramos A., Ocultamiento del mensaje de señales de vozas usando la transformada ondita de Haar discreta, Revista información tecnológica, 21(3), pp. 135-140.(2010).

Ngugi B., Kamis A. y Tremaine M., Intention to Use Biometric Systems, A Journal of Electronic Services in the Public and Private Sectors, 7(3), pp. 20 - 46, (2011).

Rubio E. y Jáuregui J., A Wavelet Approach to Estimate The Quality of Ground Parts, Journal of applied research and technology, 10(1), pp. 28-37 (2012).

Olivares-Mercado J., Sánchez-Pérez G., Nakano-Miyatake M. y Pérez-Meana H., Feature Extraction and Face Verification Using Gabor and Gaussian Mixture Models, Lecture Notes in Artificial Intelligence LNAI pp. 48-27, (2007).

Santamaría F., Cortés C. y Román F., Uso de la Transformada de Ondeletas (Wavelet Transform) en la Reducción de Ruidos en las Señales de Campo Eléctrico producidas por Rayos, Información Tecnológica, 23(1), pp. 65-78, (2012).

Turk M. y Pentland A., Eigenfaces for Recongnition, Journal of Cognitive Neuroscience, 3(1) 71-86, (1991).

Trussell, H.J., Saber E. y Vrhel M., Color image processing: basics and special issue overview, IEEE Signal Processing Magazine, ISSN 1053-5888, 22(1), pp. $14-22$, (2005).

Vezzeti E. y Marcolin F., Geometrical descriptors for human face morphological analysis and recognition, Robotics and Autonomous Systems, 60(6), pp. 928 - 939, (2012).

Yu X., Wang X., y Chen J., Support Vector Machine Regressionfor Reactivity Parameters of Vinyl monomers, Journalof the Chilean Chemical Society, 56(3), pp. 746-751, (2011).

Zhonghua L., Haixia Z., Jiexin P. y Hui W., Face recognition under varying illumination, Neural Computing and Applications, 23(1),pp. $133-139,(2013)$. 